\title{
Patient-Centric LTOT: No Room for Complacency
}

The benefits of the continuous administration of supplemental long-term oxygen therapy (LTOT) for the amelioration of the sequelae of severe chronic hypoxemia (baseline $\mathrm{P}_{\mathrm{aO}_{2}} \leq 55 \mathrm{mg} \mathrm{Hg}$ or $\mathrm{S}_{\mathrm{pO}_{2}} \leq 88 \%$ ) secondary to COPD, are well established. When properly prescribed and delivered, LTOT improves survival, increases exercise tolerance, reduces dyspnea, and improves the overall quality of life in this vulnerable patient population. ${ }^{1}$ As with any other medication prescribed for sustained symptom control of a chronic condition (eg, hypertension, hypercholesterolemia), the oxygen dose must be appropriate at all activity levels to prevent arterial oxygen desaturation on a 24/7 basis. In the words of the late Dr Tom Petty, one of the early pioneers and perhaps the strongest advocate ever of this vital, life-saving intervention, "Suffice it to say the bottom line is that adequate oxygenation is judged by pulse oximetry under conditions of rest, exercise, and during sleep." 2

What this means in practical terms is that adequate oxygen saturation must be maintained across a wide continuum of daily activities. This includes situations where systemic oxygen demand is probably at its lowest-in the resting/sedentary state - to where systemic oxygen demand may increase substantially_during exercise as well as during other activities of daily living such as walking, bathing, meal preparation, eating, light housekeeping, and during sleep. Further, the recent mandate by the Federal Aviation Administration to allow portable oxygen concentrators aboard commercial aircraft has added yet another point on the LTOT continuum: situations where decreased ambient oxygen partial pressure exists due to altitude. ${ }^{3}$ While all of the foregoing clearly imply that the oxygen dose of a particular patient should be continually adjusted in response to increased systemic demands, the reality of the matter is that the majority of LTOT users are prescribed a fixed continuous flow setting, typically $2 \mathrm{~L} / \mathrm{min}$, in spite of a growing emphasis on a "titrate to saturate" approach to optimum LTOT. ${ }^{3}$ Failure to maintain effective symptom control because of suboptimal oxygenation may lead to the perception that a patient's disease severity is worsening, when in fact the primary problem may well be the use of poorly performing oxygen dispensing equipment. ${ }^{4}$ However, there is yet another consideration: even with a traditional continuous oxygen dispensing system, oxygen dosing may be suboptimal.
In this issue of Respiratory CARE, Cirio and Nava report the results of a study of a novel automatic flow regulator that essentially converts a standard portable liquid oxygen canister, delivering a continuous liter flow, into a servocontrolled oxygen dispensing device. ${ }^{5}$ Using an integrated pulse oximeter, the flow regulator automatically adjusted the oxygen flow rate to maintain a target $\mathrm{S}_{\mathrm{pO}_{2}}$ of $94 \%$ during 15-minute constant-work-load cycling exercise tests. In this randomized crossover study, the variable was how well the flow regulator automatically responded to a fall in $\mathrm{S}_{\mathrm{pO}_{2}}$, compared to manual adjustments made by the respiratory therapist co-investigator. The device (not yet available in the U.S.) clearly proved to be far superior in terms of response time, as measured by the amount of time a subject's saturation remained below the target $\mathrm{S}_{\mathrm{pO}_{2}}$. And, as would be expected, subjects using the flow regulator exhibited a significantly higher mean $\mathrm{S}_{\mathrm{pO}_{2}}$ that when receiving manual titration.

\section{See the Original Study on Page 429}

The implications of this study are several. Cirio and Nava identified 3 results from their testing:

- Significant improvement in mean $\mathrm{S}_{\mathrm{pO}_{2}}$ during exercise testing

- Significant reduction of time spent below target $\mathrm{S}_{\mathrm{pO}_{2}}$ during exercise

- Significantly quicker response time by the device than by the respiratory therapist manually titrating oxygen flow to maintain an adequate saturation during exercise

While all 3 are important findings, the first 2 have the potential to improve patient care and reduce comorbidities associated with suboptimal LTOT. However, while manual titration by a respiratory therapist does indeed work, once a patient leaves the care of a professional clinician, titration to saturation rarely happens. This feature alone enhances the value of a device able to automatically titrate to a target saturation.

The results of a similar study, by Rice and colleagues, at the Veterans Affairs Medical Center in Minneapolis, were presented at the 2007 American Thoracic Society meeting. ${ }^{6}$ Rice et al tested a closed-loop oxygen system that used oximetry to monitor and adjust an intermittent-flow 
oxygen delivery device. Their objective was to show equivalency to other intermittent-flow devices to determine the difference in oxygen savings. Since the intermittent-flow device would turn itself off when the oxygen saturation level was adequate $(\geq 90 \%)$, the oxygen savings were substantial. The device described by Cirio and Nava has similar capabilities, although their study evaluated continuous-flow oxygen delivery.

The target saturation used by Cirio and Nava (94\%) was interesting, as there is still no consensus on the appropriate saturation to effectively maintain a patient's oxygen needs. Many clinicians feel that $\geq 90 \%$ is adequate, yet a higher saturation may be of benefit for exercise and conditioning. ${ }^{7}$ One explanation for the widespread complacency of accepting a lower target saturation may be the limited capability of certain portable home oxygen systems to deliver oxygen at a rate that provides a higher saturation, and, most importantly, when systemic oxygen demand increases.

Improvement in mean oxygen saturation during the 15minute exercise test was the primary objective of Cirio and Nava's study, and the data show a significant improvement. For many patients on LTOT, without constant clinician intervention or an automatic-adjusting oxygen system, a substantial amount of time is spent below the target $\mathrm{S}_{\mathrm{pO}_{2}}$, resulting in suboptimal therapy and associated morbidities. ${ }^{8}$ The method of automatically adjusting oxygen flow was unique to the system studied by Cirio and Nava, as the device was designed to aggressively respond to desaturation by incrementally adjusting the flow to reestablish the target $\mathrm{S}_{\mathrm{pO}_{2}}$.

Many clinicians have experienced the challenge of increasing the dose of oxygen slowly in response to a desaturation, and finding that they are always chasing the $\mathrm{S}_{\mathrm{pO}_{2}}$ to achieve the desired saturation. The study by Cirio and Nava shows that responding quickly and aggressively by increasing the flow more than the typical $1 \mathrm{~L} / \mathrm{min}$ increment can maintain adequate oxygen saturation, and that a slow reduction in oxygen dose, as the saturation stabilizes, can establish an improved mean saturation over a period of time.

Cirio and Nava's study, even though it was a small evaluation of a novel oxygen delivery system, highlights some important issues in LTOT. The most interesting and

The authors have disclosed no conflicts of interest.

Correspondence: Patrick J Dunne MEd RRT FAARC, HealthCare Productions, Sunny Hills Station, PO Box 5767, Fullerton CA 92838-9998. E-mail: pjdunne@sbcglobal.net.

DOI: $10.4187 /$ respcare. 01288 noteworthy is identifying the need to adjust the oxygen flow consistently in the face of changing demand. Patients are always changing work loads, so oxygen needs to be delivered to meet their clinical requirements. The traditional approach of testing at a resting level and setting a fixed oxygen prescription is still a very common practice. Titrating with exercise is becoming more prevalent, using the standard 6-minute walk test. With one fixed resting prescription and one fixed exercise prescription, patients still do not benefit from traditional oxygen systems that do not adjust to their ongoing and ever-changing oxygen needs.

Although the majority of currently available portable home oxygen technology is fixed in its delivery capability, patients are dynamic in their activity and oxygen needs. The study by Cirio and Nava addresses the most critical aspect of LTOT: the continual delivery of an effective dose of a vital controller medication, at a therapeutic level, in response to the patient's ever-changing needs. This is truly patient-centric LTOT at its best.

Patrick J Dunne MEd RRT FAARC HealthCare Productions Fullerton, California

Robert W McCoy RRT FAARC Valley Inspired Products Apple Valley, Minnesota

\section{REFERENCES}

1. Cranston JM, Crockett A, Moss J, Alpers JH. Domiciliary oxygen for chronic obstructive pulmonary disease. Cochrane Database Syst Rev 2005;(4):CD001744.

2. Petty TL, McCoy R, Nett L, Bowden K. Adventures of an oxy-phile, 2nd edition. Denver: Snowdrift Pulmonary Conference; 2010.

3. Dunne PJ. The clinical impact of new long-term oxygen therapy technology. Respir Care 2009;54(8):1100-1111.

4. McCoy RW, Carlin BW. Product performance variability with home portable oxygen systems may impact patient performance outcomes: it may be the device, not the disease (editorial). Respir Care 2009; 54(3):324-326.

5. Cirio S, Nava S. Pilot study of a new device to titrate oxygen flow in hypoxic patients on long-term oxygen therapy. Respir Care 2011; 56(4):429-434.

6. Rice KL, Schmidt MF, Buan JS, Lebahn F, Schwarzrock TK. A portable closed-loop oxygen conserving device for stable COPD patients: comparison with fixed dose delivery systems (abstract). Am J Respir Crit Care 2007;175(Suppl):A135.

7. Emtner M. Porszasz J. Burns M, Somfay A, Casaburi R. Benefits of supplemental oxygen in exercise training in nonhypoxemic chronic obstructive pulmonary disease patients. Am J Respir Crit Care Med 2003; 168(9):1034-1042.

8. Sliwinski P, Lagosz M, Górecka D, Zielinski. The adequacy of oxygenation in COPD patients undergoing long-term oxygen therapy assessed by pulse oximetry at home. Eur Respir J 1994;7(2):274278. 